\title{
Size dependence of the optical and thermal properties of sodium clusters $\left.{ }^{*}\right)$
}

\author{
H. HABERLAND \\ Fakultät für Physik, Universität Freiburg - D-79104 Freiburg, Germany
}

(ricevuto il 3 Settembre 1997; approvato il 15 Ottobre 1997)

PACS 36.40 - Atomic and molecular clusters.

PACS 01.30.Cc - Conference proceedings.

The transition from the atom, molecule, over the cluster, to the solid can be very well studied for sodium. Only for two cases does the optical response show broad plasmon-like peaks which are well described by the "Nearly Free Electron" or jellium model: a) for clusters with more than about 20 atoms, and b) for small $(7 \leq n \leq 20)$ liquid clusters. Only for these two cases is a comparison to the nuclear physics case meaningful.

Sharp "molecular-like" peaks are observed for small, cold sodium clusters. The slow transition between these only seemingly different results was shown, and it was discussed how the cluster temperature influences this transition [1,2].

Three other topics were briefly mentioned: 1) first measurements of the melting point and latent heat of fusion of free, size selected clusters, 2) the multiple plasmon excitation of a size selected cluster with an intense femto-second laser pulse, and 3) a technological application of clusters to thin film formation [3].

\section{REFERENCES}

[1] Ellert Ch., Schmidt M., Schmitt Ch., Reiners Th. and Haberland H., Phys. Rev. Lett., 75 (1995) 1731.

[2] Reiners Th., Ellert Ch., Schmidt M.and Haberland H., Phys.Rev.Lett., 74 (1995) 1558.

[3] Haberland H., Mall M., Moseler M., Qiang Y., Reiners Th. and Thurner Y., J. Vac. Sci. Technol. A, 12 (1994) 2925; Haberland H., Insepov Z. and Moseler M., Phys. Rev. B, 51 (1995) 11061.

$\left({ }^{*}\right)$ Paper presented at the 174. WE-Heraeus-Seminar "New Ideas on Clustering in Nuclear and Atomic Physics", Rauischholzhausen (Germany), 9-13 June 1997. 